\title{
A study revealing the key aroma compounds of steamed bread made by Chinese traditional sourdough ${ }^{*}$
}

\author{
Guo-hua ZHANG ${ }^{1}$, Tao $\mathrm{WU}^{2}$, Faizan A. SADIQ ${ }^{3,4}$, Huan-yi $\mathrm{YANG}^{3,4}$, \\ Tong-jie LIU $^{3,4}$, Hui RUAN ${ }^{3,4}$, Guo-qing $\mathrm{HE}^{\dagger \ddagger 3,4}$ \\ ( ${ }^{1}$ College of Life Science, Shanxi University, Taiyuan 030006, China) \\ $\left({ }^{2}\right.$ College of Food Engineering and Biotechnology, Tianjin University of Science \& Technology, Tianjin 300457, China) \\ $\left({ }^{3}\right.$ College of Biosystems Engineering and Food Science, Zhejiang University, Hangzhou 310058, China) \\ ( ${ }^{4}$ Zhejiang Provincial Key Laboratory of Food Microbiology, Zhejiang University, Hangzhou 310058, China) \\ †E-mail: gqhe@zju.edu.cn \\ Received Mar. 23, 2016; Revision accepted June 23, 2016; Crosschecked Sept. 11, 2016
}

\begin{abstract}
Aroma of Chinese steamed bread (CSB) is one of the important parameters that determines the overall quality attributes and consumer acceptance. However, the aroma profile of CSB still remains poorly understood, mainly because of relying on only a single method for aroma extraction in previous studies. Therefore, the objective of this study was to determine the volatile aroma compounds of five different samples of CSB using three different aroma extraction methods, namely solid-phase microextraction (SPME), simultaneous distillation-extraction (SDE), and purge and trap (P\&T). All samples showed a unique aroma profile, which could be attributed to their unique microbial consortia. (E)-2-Nonenal and $(E, E)-2,4-$ decadienal were the most prevalent aromatic compounds revealed by SDE, which have not been reported previously, while ethanol and acetic acid proved to be the most dominant compounds by both SPME and P\&T. Our approach of combining three different aroma extraction methods provided better insights into the aroma profile of CSB, which had remained largely unknown in previous studies.
\end{abstract}

Key words: Steamed bread, Sourdough, Simultaneous distillation-extraction (SDE), Solid-phase microextraction (SPME), Purge and trap (P\&T)

http://dx.doi.org/10.1631/jzus.B1600130

CLC number: R15

\section{Introduction}

Chinese steamed bread (CSB), also known as mantou, is a Chinese traditional fermented product that has been enjoying great popularity among the Chinese people since its origin over 1500 years ago. It is a most widely consumed fermented wheat flour product in China and accounts for around $40 \%$ of the total wheat consumption (He et al., 2003; Kim et al.,

\footnotetext{
Corresponding author

* Project supported by the National Natural Science Foundation of China (No. 31371826) and the China Postdoctoral Science Foundation (No. 2016M592002)

(Di) ORCID: Guo-qing HE, http://orcid.org/0000-0002-1177-8016

(C) Zhejiang University and Springer-Verlag Berlin Heidelberg 2016
}

2009). It is formulated with wheat flour, water, and a leavening agent, which may be either yeast or traditional sourdough. The use of sourdough for the leavening of steamed bread is one of the ancient biotechnological processes in the field of cereal fermentation. Despite the ease of using baker's yeast, the use of sourdough is still preferred over it because of the great benefits being associated with the use of sourdough. It is well established that bread leavened by sourdough has an improved taste and a delicate texture, better aromatic profile, and longer shelf-life compared to conventional bread (Arendt et al., 2007; Katina and Poutanen, 2013). There are also various health benefits associated with sourdough. For instance, it is known that the metabolites and various 
enzymes produced by lactic acid bacteria (LAB) during sourdough-based fermentation help to reduce postprandial hyperglycemia and phytate lever (Galle and Arendt, 2014), and, on the other hand, increase mineral bioavailability and exopolysaccharide production with prebiotic activity (Arendt et al., 2007).

The role of microbes in conferring aromatic properties to fermented cereal products is well known (Salim-ur-Rehman et al., 2006) and the complex microbial ecology of Chinese traditional sourdough has been studied in detail by us (Zhang and $\mathrm{He}, 2013$; Zhang et al., 2015) and other researchers (Luangsakul et al., 2009; Zhang et al., 2011). Because of high importance of CSB in dietary life style, there is a great deal of interest among food technologists to study and investigate its aromatic compounds. The role of sourdough in developing aromatic compounds in wheat bread is well acknowledged (Lorenz and Kulp, 2003). The potential of individual strains of yeast (Saccharomyces cerevisiae and Candida guilliermondii) and LAB (such as Lactobacillus plantarum) to produce aromatic compounds during sourdough fermentation has been explored (Stolz et al., 1993). Similarly, volatile profiles of French sourdoughs (Frasse et al., 1993), spontaneously fermented sourdoughs (Kim et al., 2009; Kam et al., 2011), as well as of bread leavened by mixed cultures of yeasts and LAB (Lactobacillus delbrueckii ssp. Bulgaricus, or Lactobacillus helveticus and Kluyveromyces marxianus) have been studied (Plessas et al., 2008). Wu et al. (2012) studied the aromatic compounds derived from spontaneously fermented and LAB-fermented steamed breads using gas chromatography/mass spectroscopy (GC/MS) using only one flavor extraction technique, namely solid-phase microextraction (SPME). Nevertheless, there is a paucity of information concerning the aroma profile of the breads leavened by Chinese traditional sourdoughs.

It is well established that different extraction methods for volatile aroma compounds have several advantages and disadvantages (Etievant, 1996). Therefore, it is necessary to use many extraction methods together in order to have a real picture of the aroma profile (Mamede and Pastore, 2006), as there is no single standard method that accurately reflects the aroma profile of food (Reineccius, 2005). These methods have their unique advantages in extracting the volatile aromatic compounds of food. Simultaneous distillation-extraction (SDE), for instance, is very efficient for the recovery of aromatic compounds with medium to high boiling points. Purge and trap (P\&T) is a dynamic head technique that allows the successful extraction and concentration of highly volatile compounds. It purges the samples in the presence of an inert gas, reflecting the way one breathes in the aroma of food, and allows a sensible correlation with sensory studies (Mamede and Pastore, 2006). SPME is a very simple, rapid, and cost-effective method that allows sampling, extraction, and concentration in a single solvent-free step (Vas and Vékey, 2004). A broad range of aromatic compounds were obtained in a Korean fermented red pepper paste with the combined use of these three methods (Kang and Baek, 2014). Therefore, the objective of this work was to study the volatile aromatic compounds of CSB made by traditional sourdough samples collected from different areas of China, using three different extraction methods, namely, SPME, P\&T, and SDE.

\section{Materials and methods}

\subsection{Wheat flour and sourdough samples}

In this study, wheat flour with low gluten content was used for making CSB and was bought from a local supermarket. The protein, moisture, wet gluten, and ash contents of the wheat flour were $7.56 \%$, $12.57 \%, 18.50 \%$, and $0.05 \%$, respectively.

Chinese traditional sourdough samples were aseptically collected from private households and steamed-bread shops from different provinces of China. They were named as Hn (from Henan Province), Sx (from Shanxi Province), Gs (from Gansu Province), Hf (from Anhui Province), and $\mathrm{Hr}$ (from Heilongjiang Province). The samples were stored at $4{ }^{\circ} \mathrm{C}$ during transportation.

\subsection{Fermentation of steamed bread by sourdough}

First, $140 \mathrm{~g}$ wheat flour was mixed with $20 \mathrm{~g}$ of each traditional sourdough sample and $70 \mathrm{ml}$ distilled/ deionized (dd) water for 5-10 min. Mixing was followed by fermentation at $(28 \pm 2)^{\circ} \mathrm{C}$ and $75 \%$ relative humidity in a controlled fermentation cabinet (LX$\mathrm{X} 24$, Shandong, China). Second, $60 \mathrm{~g}$ wheat flour and $20 \mathrm{ml}$ dd water were remixed with the full fermented dough for $15 \mathrm{~min}$. Then the dough was rolled into round shape manually and fermented at $(28 \pm 2)^{\circ} \mathrm{C}$ and $75 \%$ relative humidity for $40 \mathrm{~min}$ in a fermentation 
cabinet. Third, the proofed dough samples were steamed for $30 \mathrm{~min}$ in a steamer (Joyong, Hangzhou, China). The control sample was prepared in the same way as described for sourdough samples, but without any leavening agent.

\subsection{Extraction methods of volatile compounds}

\subsubsection{SDE method}

Volatile compounds of the steamed bread samples including the control ( $30 \mathrm{~g}$ steamed bread crumbs plus $300 \mathrm{ml}$ distilled water) were extracted for $2.5 \mathrm{~h}$ by $30 \mathrm{ml}$ dichloromethane in an SDE apparatus (Kontes Co., Ltd., USA). The solvent-phase extract was treated with anhydrous $\mathrm{Na}_{2} \mathrm{SO}_{4}$ and concentrated to $1 \mathrm{ml}$ at $40{ }^{\circ} \mathrm{C}$ using a Vigreux column $(30 \mathrm{~cm} \times$ $2 \mathrm{~cm}$ ), and then stored at $-80^{\circ} \mathrm{C}$ for further analysis.

\subsubsection{SPME method}

Several parameters (fiber choice, extraction time, and extraction temperature) influencing the SPME analyses were studied. The optimal parameters were arrived at as follows: $1.5 \mathrm{~g}$ of steamed bread crumbs was placed in 15-ml vials for each analysis. SPME was performed with the SPME fiber assembly carboxen/polydimethylsiloxane (CAR/PDMS, $75 \mu \mathrm{m})$ mounted on an SPME manual holder assembly (Supelco, Bellefonte, USA). The vials containing the samples were placed in a water bath $\left(75^{\circ} \mathrm{C}\right)$ for $30 \mathrm{~min}$ to equilibrate, and then the septum was pierced with the SPME needle. The fiber was exposed to the headspace of the sample for $30 \mathrm{~min}$, and after the extraction time the fiber was retracted into the needle and transferred immediately into the injection port of a gas chromatograph and desorbed for $10 \mathrm{~min}$ at $250{ }^{\circ} \mathrm{C}$ for further analysis.

\subsubsection{P\&T method}

Two grams of steamed bread crumbs were taken in a fritted sparge tube and heated at $50{ }^{\circ} \mathrm{C}$. The volatile components were captured using a Stratum P\&T concentrator (Tekmar, Ohio, USA) according to the method of Wang et al. (2012).

\subsection{Gas chromatography-olfactometry-mass spec- trometer (GC-O-MS) analysis}

The analyses of volatiles were performed on a GC apparatus (7890A, Agilent, Palo Alto, CA, USA) coupled to a Triple Quad 7000B (Agilent), and equipped with a Sniffer 9000 olfactometer (Gerstel, $\mathrm{KG}$, Germany). Separations in GC were performed on a DB-Wax column $(30 \mathrm{~m} \times 0.320 \mathrm{~mm}$ i.d., $0.25 \mu \mathrm{m}$ film thickness; J\&W Scientific, USA). The carrier gas used was ultrahigh-purity helium, and the flow rate was $1.0 \mathrm{ml} / \mathrm{min}$.

The oven temperature was programmed at $40{ }^{\circ} \mathrm{C}$ and held for $3 \mathrm{~min}$, then increased at $5{ }^{\circ} \mathrm{C} / \mathrm{min}$ to $200{ }^{\circ} \mathrm{C}$, and then increased at $10{ }^{\circ} \mathrm{C} / \mathrm{min}$ to $230^{\circ} \mathrm{C}$, where it was held for $5 \mathrm{~min}$. The temperatures of the injector and the GC/MS interface were $250{ }^{\circ} \mathrm{C}$ and $280{ }^{\circ} \mathrm{C}$, respectively. Electron-impact mass spectra were generated at $70 \mathrm{eV}$, with $\mathrm{m} / \mathrm{z}$ (mass-to-charge ratio) scan ranging from 35 to $550 \mathrm{amu}$ (atomic mass unit), and the ion source temperature of $230^{\circ} \mathrm{C}$.

GC-O was performed by three experienced panelists (Wang et al., 2012).

\subsection{Compound identification}

The identification of volatile compounds was based on comparing the mass spectra and retention indices (RIs) with reference mass spectra. The RI values and odor descriptions on DB-Wax column were matched with those of RIs having the same or similar odor quality and RI, previously reported in http://www.odour.org.uk. n-Alkanes $\left(\mathrm{C}_{7}-\mathrm{C}_{22}\right)$ were analyzed under the same conditions to calculate LRIs: $\mathrm{LRI}=100 N+100 n\left(t_{\mathrm{Ra}}-t_{\mathrm{R} N}\right) /\left(t_{\mathrm{R}(N+n)}-t_{\mathrm{R} N}\right)$, where $N$ is the carbon number of the lower alkane and $n$ is the difference in carbon number of the two $n$-alkanes that bracket the compound; $t_{\mathrm{Ra}}, t_{\mathrm{R} N}$, and $t_{\mathrm{R}(N+n)}$ are the retention time of unknown (or test) compound, the lower alkane, and the upper alkane, respectively (van den Dool and Kratz, 1963).

\section{Results and discussion}

\subsection{General discussion}

Volatile flavor compounds from CSBs were extracted by SDE, SPME, and P\&T methods followed by their identification using GC-MS coupled with an olfactometric detector. A unique volatile aroma profile for each sample (including the control) was obtained using the three methods of extraction. This variation among the samples may be attributed to their unique microbial profile. The generation of volatile compounds in bread is evidently influenced by the metabolic activities of $\mathrm{LAB}$ and yeasts in 
sourdough and the relatively high content of aromatic compounds reported during microbial fermentation of sourdoughs (Hansen and Hansen, 1994; Hansen and Schieberle, 2005). For instance, it is known that the products dominated by homofermentative LAB are rich in diacetyls and carbonyls, whereas heterofermentative $\mathrm{LAB}$ products are rich in aldehydes, alcohols, and ethyl acetate (Spicher et al., 1982). Similarly, volatiles in yeast products are dominated by alcohols, esters, and some carbonyl compounds (Damiani et al., 1996).

In our previous work, we reported the microbiota of these five sourdough samples by culture-dependent and culture-independent (polymerase chain reactiondenaturing gradient gel electrophoresis (PCR-DGGE) and high-throughput sequencing) techniques, and concluded that there existed remarkable variation among the microbiota present in the samples (Zhang and He, 2013; Zhang et al., 2015). The variation in microflora is manifested in terms of different aroma profiles of each sample. However, it is also important to mention that the aroma of a bread crumb is also affected by many factors like the wheat variety (Starr et al., 2015). We were unable to ensure that all the sourdough samples were made from one kind of wheat flour. It is equally important to highlight that several aromatic compounds are produced as a result of enzymatic or autoxidation of several wheat flour fractions. For instance, the production of aldehydes, apart from their production by microbial metabolic activities, can be a result of oxidation of the lipid fraction of wheat (Mann and Morrison, 1975; Frankel, 1982). Also, different parameters like dough consistency and fermentation temperature may have influence on the overall aroma profile of bread (Lorenz and Kulp, 2003). In the following sections, each volatile aroma extraction method is discussed in detail.

\subsection{Evaluation of SDE method}

An SDE method was employed to extract the volatile compounds from the samples including the control bread. Following the extraction, 19 volatile compounds were identified using GC coupled with an olfactometric detector. Results of the identification based on olfactory and mass spectral analysis are shown in Table 1. Except for 2-pentylfuran, an organic

Table 1 GC-O-MS identification of traditional sourdough steamed bread obtained by SDE method

\begin{tabular}{|c|c|c|c|c|c|c|c|c|}
\hline \multirow{2}{*}{ Compound } & \multirow{2}{*}{$\mathrm{RI}^{\mathrm{a}}$} & \multicolumn{6}{|c|}{ Relative peak area (\%) } & \multirow{2}{*}{ Odor $^{b}$} \\
\hline & & Control & $\mathrm{Hr}$ & $\mathrm{Sx}$ & $\mathrm{Hn}$ & Gs & $\mathrm{Hf}$ & \\
\hline \multicolumn{9}{|l|}{ Aldehydes } \\
\hline Hexanal & 1081 & - & 10.12 & 25.09 & 11.19 & 14.76 & 4.96 & Green \\
\hline Heptanal & 1183 & 37.13 & 9.46 & 9.57 & 13.66 & 13.75 & 4.94 & Planty green \\
\hline Octanal & 1287 & - & - & - & - & 4.17 & - & Pungent \\
\hline (E)-2-Heptenal & 1381 & - & 3.43 & - & - & - & - & Fatty \\
\hline Nonanal & 1392 & - & 2.90 & 2.10 & 3.99 & 2.88 & 1.16 & Slightly fatty \\
\hline (E)-2-Octenal & 1425 & 31.50 & 13.17 & 9.74 & 9.25 & 10.54 & 4.63 & Nutty \\
\hline Benzaldehyde & 1515 & - & - & - & 1.14 & - & 0.52 & Bitter almond \\
\hline (E)-2-Nonenal & 1532 & 11.53 & 10.63 & 6.31 & 8.23 & 6.58 & 4.00 & Cucumber \\
\hline (E)-2-Decenal & 1642 & - & 7.12 & 3.37 & 9.69 & 2.52 & 3.82 & Fatty \\
\hline$(E, E)-2,4$-Nonadienal & 1700 & - & 6.06 & 2.12 & - & 1.93 & 3.22 & Fruity \\
\hline 2-Undecenal & 1750 & - & 8.05 & 3.70 & 8.00 & 2.07 & 4.67 & Bitter \\
\hline$(E, E)$-2,4-Decadienal & 1807 & 19.84 & 8.74 & 22.87 & 12.84 & 11.34 & 34.45 & Fried potato \\
\hline Total & & 100.00 & 79.68 & 84.87 & 77.99 & 70.54 & 66.37 & \\
\hline \multicolumn{9}{|l|}{ Alcohols } \\
\hline 1-Pentanol & 1252 & - & - & - & 8.60 & - & 7.81 & Wax \\
\hline 1-Hexanol & 1361 & - & - & - & 4.34 & - & 14.79 & - \\
\hline 1-Octen-3-ol & 1445 & - & - & - & - & - & 0.94 & Raw mushrooms \\
\hline Total & & 0.00 & 0.00 & 0.00 & 12.94 & 0.00 & 23.54 & \\
\hline \multicolumn{9}{|l|}{ Ketones } \\
\hline 2-Heptanone & 1180 & - & 3.18 & - & - & 6.16 & - & Cheese \\
\hline 2-Octanone & 1283 & - & 6.34 & 5.39 & 3.84 & 8.47 & 3.29 & Fruity \\
\hline 2-Nonanone & 1387 & - & 2.71 & 1.94 & 0.76 & 1.70 & 0.48 & Blue cheese \\
\hline Total & & 0.00 & 12.23 & 7.33 & 4.60 & 16.33 & 3.77 & \\
\hline \multicolumn{9}{|l|}{ Others } \\
\hline 2-Pentylfuran & 1231 & - & 8.09 & 7.81 & 4.46 & 13.12 & 6.34 & Fruity, green \\
\hline Total & & 0.00 & 8.09 & 7.81 & 4.46 & 13.12 & 6.34 & \\
\hline
\end{tabular}

${ }^{a}$ Retention indices on DB-Wax column; ${ }^{\mathrm{b}}$ Odor perceived at the sniffing port 
heteroaromatic compound previously reported in yeast extract (Mahadevan and Farmer, 2006), the rest of the volatile compounds were aldehydes (12), alcohols (3), and ketones (3). The data regarding the volatile compounds extracted and identified based on this method were subjected to the principal component analysis (PCA), as shown in Fig. 1. The Hf sample was different from the others due to the presence of high contents of $(E, E)$-2,4-decadienal, which is considered a key aromatic compound in wheat bread crumb. The presence of this odorant highly depends on the strains used and the fermentation conditions (Vermeulen et al., 2007). Some aldehydes (hexanal, heptanal, octanal, nonanal) as determined by SDE can be the result of increased activity of oxidative yeasts (Lorenz and Kulp, 2003).

Sourdough-fermented samples produced more aromatic compounds as compared to the control. For instance, in the control bread, there were only 4 aromatic compounds (aldehydes, namely heptanal, $(E)$ 2-octenal, $(E)$-2-nonenal, and ( $E, E)$-2,4-decadienal), compared to the 19 odorants (12 aldehydes, 3 alcohols, 3 ketones, and 12-pentylfuran) in the bread fermented with sourdough. Also, great inter-sample variation in the aroma profile was also observed. As shown in Table 1, the relative proportion of aldehydes is different in five sourdough breads, ranging from $66.37 \%$ (Hf sample) to $84.87 \%$ (Sx sample). Similarly, the ketone content also showed variation among the samples, with the dominance of 2-octanone. Alcohols were only detected in $\mathrm{Hn}$ and Hf samples, $12.94 \%$ and $23.54 \%$, respectively. The highest level of hexanal (25.09\%) was found in the Sx sample and, similarly, the highest contents of $(E)$-2-nonenal and $(E, E)-2,4$-decadienal were presented in $\mathrm{Hr}$ and $\mathrm{Hf}$ samples, $10.63 \%$ and $34.45 \%$ respectively. It can be seen in Fig. 2 that the highest content of aldehydes was detected by using SDE method. A comparison of all the methods used for the extraction of aromatic compounds is given in a later section. To the best of our knowledge, the three aldehydes $((E)$-2-decenal, $(E, E)$-2-4-nonadienal, and 2-undecenal) and the two ketones (2-heptanone and 2-nonanone) have not been previously reported in CSBs.

Aldehydes are assumed to be generated by enzymatic oxidation of linoleic acids through the Ehrlich pathway by the degradation of certain amino acids during dough mixing and fermentation (Schieberle, 1996). (E,E)-2,4-Decadienal and $(E)-2-$ nonenal were reported as the key aroma constituents in wheat bread crumb (Vermeulen et al., 2007). Hydrogen peroxide formation by some lactobacilli and $S$. cerevisiae can enhance lipid oxidation to promote the oxidation of linoleic acid to $(E, E)$-2,4-decadienalin in wheat dough (Liao et al., 1998), resulting in increased levels of aroma compounds. Heterofermentative lactobacilli can rapidly reduce aldehydes $((E, E)$-2,4-decadienal and $(E)$-2-nonenal) to their corresponding alcohols, whereas $S$. cerevisiae shows a comparatively low activity. $S$. cerevisiae can reduce both the aldehyde moiety and the carboncarbon double bond, thus resulting in the production of saturated alcohols. Unsaturated aldehydes derived from lipid oxidation play a dominant role in the aroma of many fermented foods (Vermeulen et al., 2007).
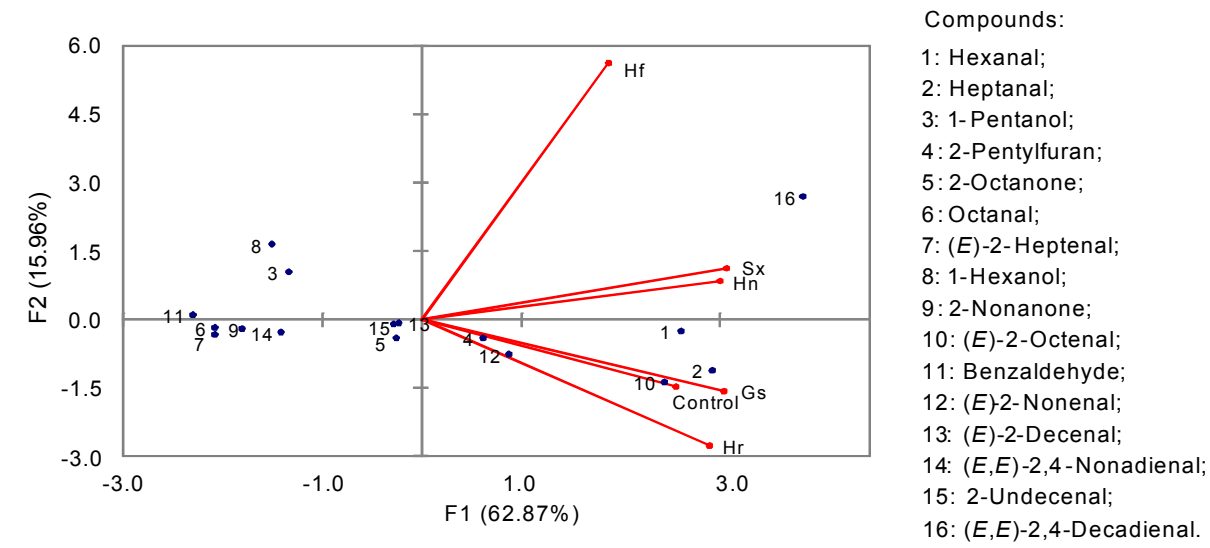

Fig. 1 Principal component analysis of volatiles of traditional sourdough steamed bread obtained by SDE method 


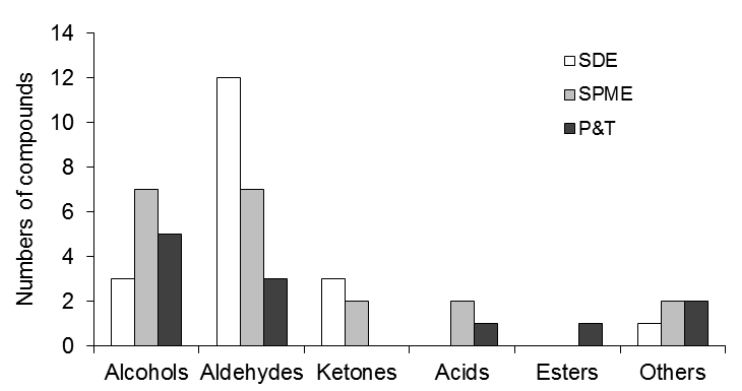

Fig. 2 Comparison of the compounds identified by the SDE, SPME, and P\&T

\subsection{SPME}

A total of 20 volatile compounds were identified by GC-O-MS (Table 2). Apart from two compounds (2-pentylfuran and naphthalene), the rest of the volatile compounds extracted by SPME were identified as aldehydes (7), alcohols (7), acids (2), and ketones (2). PCA of the odorants extracted by SPME method is shown in Fig. 3. Ethanol, acetic acid, and 2pentylfuran clustered the samples into three groups: group 1, control bread; group 2, Sx and Hf samples; and group 3, Gs, Hn, and $\mathrm{Hr}$ samples.

Table 2 GC-O-MS identification of volatiles of traditional sourdough steamed bread obtained by SPME method

\begin{tabular}{|c|c|c|c|c|c|c|c|c|}
\hline \multirow{2}{*}{ Compound } & \multirow{2}{*}{$\mathrm{RI}^{\mathrm{a}}$} & \multicolumn{6}{|c|}{ Relative peak area (\%) } & \multirow{2}{*}{ Odor $^{\mathrm{b}}$} \\
\hline & & Control & $\mathrm{Hr}$ & Sx & $\mathrm{Hn}$ & Gs & Hf & \\
\hline \multicolumn{9}{|l|}{ Aldehydes } \\
\hline (E)-2-Butenal & 1028 & - & 0.91 & - & - & - & - & - \\
\hline Hexanal & 1081 & 5.78 & 1.39 & 1.94 & 2.95 & 2.96 & 2.13 & Green \\
\hline Heptanal & 1183 & - & - & - & 0.64 & - & - & Planty green \\
\hline (E)-2-Heptenal & 1318 & 1.06 & - & 1.21 & 1.04 & 1.69 & 0.89 & Fatty \\
\hline Nonanal & 1392 & - & - & 0.41 & 1.02 & 1.70 & 0.69 & Slightly floral \\
\hline Furfural & 1460 & - & 2.05 & 7.81 & 2.22 & 4.13 & 6.49 & - \\
\hline Benzaldehyde & 1515 & 13.27 & 6.78 & 9.38 & 8.56 & 4.62 & 5.83 & Bitter almond \\
\hline Total & & 20.11 & 11.13 & 20.75 & 16.43 & 15.10 & 16.03 & \\
\hline \multicolumn{9}{|l|}{ Alcohols } \\
\hline Ethanol & 925 & - & 32.40 & 9.15 & 18.01 & 13.19 & 11.72 & Alcoholic, strong \\
\hline 3-Methyl-1-butanol & 1198 & - & 2.13 & 1.30 & 8.25 & - & - & Sweet, green \\
\hline 1-Pentanol & 1247 & 7.01 & 1.05 & 1.27 & 1.00 & 4.26 & 1.71 & Alcoholic \\
\hline 1-Hexanol & 1361 & 19.71 & 26.52 & 28.29 & 15.12 & 25.74 & 36.89 & - \\
\hline 1-Heptanol & 1448 & - & - & 3.99 & - & 4.35 & - & - \\
\hline 1-Octanol & 1551 & 1.42 & 1.32 & 2.17 & 0.41 & 1.69 & 1.85 & - \\
\hline Phenylethyl alcohol & 1903 & - & 3.06 & 3.38 & 27.58 & 7.96 & 2.95 & - \\
\hline Total & & 28.14 & 66.48 & 49.55 & 70.37 & 57.19 & 55.12 & \\
\hline \multicolumn{9}{|l|}{ Ketones } \\
\hline 2-Pentanone & 978 & - & 0.63 & - & - & - & - & Fruity \\
\hline 3-Hydroxy-2-butanone & 1292 & - & 1.22 & - & - & 2.91 & - & - \\
\hline Total & & 0.00 & 1.85 & 0.00 & 0.00 & 2.91 & 0.00 & \\
\hline \multicolumn{9}{|l|}{ Acids } \\
\hline Acetic acid & 1449 & - & 9.02 & 14.03 & 4.60 & 18.84 & 18.17 & Vinegar, acid \\
\hline Hexanoic acid & 1855 & - & 0.90 & 4.85 & 0.41 & 0.97 & 1.56 & - \\
\hline Total & & 0.00 & 9.92 & 18.88 & 5.01 & 19.81 & 19.73 & \\
\hline \multicolumn{9}{|l|}{ Others } \\
\hline 2-Pentylfuran & 1231 & 43.95 & 9.68 & 9.14 & 4.88 & 3.77 & 9.12 & Fruity, green \\
\hline Naphthalene & 1740 & 7.80 & 0.95 & 1.37 & 2.46 & 1.23 & - & Camphoric \\
\hline Total & & 51.75 & 10.63 & 10.51 & 7.34 & 5.00 & 9.12 & \\
\hline
\end{tabular}

${ }^{\mathrm{a}}$ Retention indices on DB-Wax column; ${ }^{\mathrm{b}}$ Odor perceived at the sniffing port 

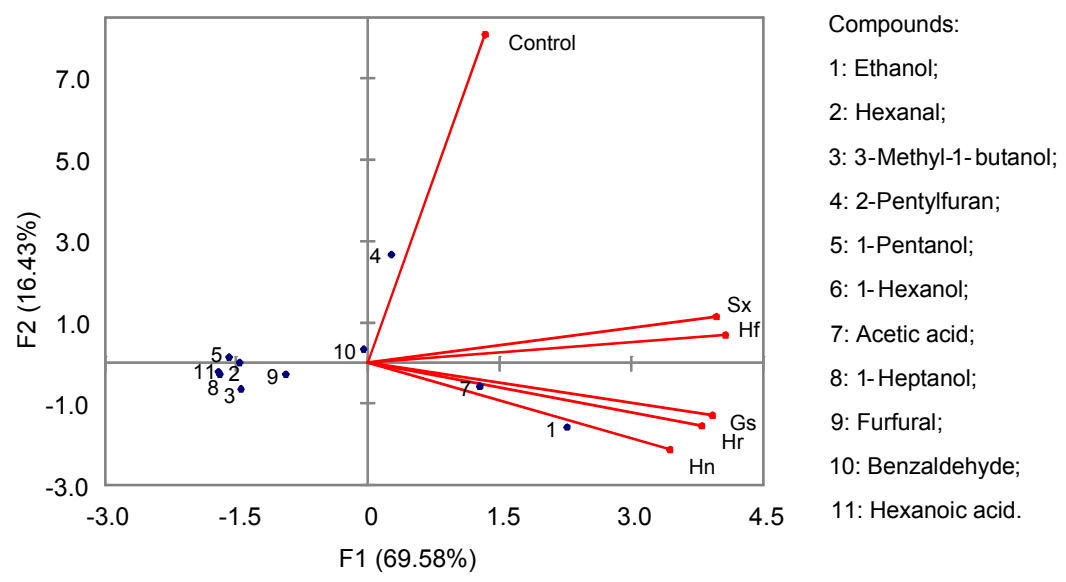

Fig. 3 Principal component analysis of volatiles of traditional sourdough steamed bread obtained by SPME method

Among the aldehydes, benzaldehyde followed by hexanal was the most abundant aldehyde in all samples including non-sourdough samples. The presence of these two compounds in sourdough breads has previously been reported (Wu et al., 2012). The production of hexanal is usually a characteristic feature of homofermentative lactobacilli. The strains of $L$. plantarum have the most homofermentative behavior among the other lactobacilli strains (Lorenz and Kulp, 2003).

In control bread, eight compounds (hexanal, (E)-2-heptenal, benzaldehyde, 1-pentanol, 1-hexanol, 1-octanol, 2-pentylfuran, and naphthalene) were identified. 2-Pentylfuran, 1-hexanol, and benzaldehyde were main compounds with the relative proportion of $43.95 \%, 19.71 \%$, and $13.27 \%$, respectively.

In sourdough steamed bread samples, alcohols were abundant, with their relative proportion ranging from $49.55 \%$ (Sx sample) to $70.37 \%$ (Hn sample). Among the alcohols, 1-hexanol and ethanol were the most abundant compounds. These two compounds have previously been reported in sourdough breads as abundant (Wu et al., 2012). 3-Methyl-1-butanol is a major volatile compound in yeast bread (Salim-urRehman et al., 2006) and was detected in $\mathrm{Hr}(2.13 \%)$, Sx $(1.30 \%)$, and Hn samples $(8.25 \%)$. A low concentration of this alcohol was found in sourdough bread; however, it could not be detected in control bread samples. Damiani et al. (1996) reported a very low concentration of this compound in spontaneously fermented sourdough breads, while a high concentration was reported in yeast-fermented breads.
Although seven aldehydes were detected by SPME, the levels of aldehydes were much lower than those of alcohols. Hexanal, furfural, and benzaldehyde were found in all sourdough bread samples. (E)-2-Heptenal and nonanal were detected in all other samples than the $\mathrm{Hr}$ sample. Apart from aldehydes and alcohols, the levels of acids were also different among the different samples, particularly acetic acid. Acetic acid, which is mainly produced by LAB (Martínez-Anaya et al., 1994; Gobbetti et al., 1995), not only exerts strong influence on the bread's flavor but also turns the environment acidic, which serves as a hurdle against the growth of moulds and ropeproducing bacilli (Rosenquist and Hansen, 1998).

\subsection{P\&T}

Twelve compounds were identified by P\&TGC/MS and GC-O methods. The volatile compounds were identified as alcohols (five compounds), aldehydes (three compounds), and others (Table 3). In addition to acetic acid (hexyl ester), compounds under the title "others" were also extracted by the SPME method. The PCA of the volatiles extracted by P\&T is shown in Fig. 4.

In control bread, six compounds (1-hexanol, hexanal, furfural, benzaldehyde, acetic acid, and 2-pentylfuran) were identified by this method. Some compounds ((E)-2-heptenal, 1-pentanol, 1-hexanol, and 1-octanol) present in control bread were detected by the SPME method but not by P\&T.

By using this method, alcohols were found to be the most abundant volatile compounds, ranging from 
Table 3 GC-O-MS identification of volatiles of traditional sourdough steamed bread obtained by P\&T method

\begin{tabular}{|c|c|c|c|c|c|c|c|c|}
\hline \multirow{2}{*}{ Compound } & \multirow{2}{*}{$\mathrm{RI}^{\mathrm{a}}$} & \multicolumn{5}{|c|}{ Relative peak area (\%) } & \multirow[b]{2}{*}{ Hf } & \multirow{2}{*}{ Odor $^{\mathrm{b}}$} \\
\hline & & Control & $\mathrm{Hr}$ & Sx & $\mathrm{Hn}$ & Gs & & \\
\hline \multicolumn{9}{|l|}{ Alcohols } \\
\hline Ethanol & 925 & - & 4.53 & 5.55 & 8.87 & 2.85 & 6.28 & Alcoholic \\
\hline 3-Methyl-1-butanol & 1198 & - & - & 25.73 & - & 72.67 & 17.50 & Sweet, green \\
\hline 1-Pentanol & 1247 & - & 27.57 & 3.22 & 71.67 & - & - & Alcoholic \\
\hline 1-Hexanol & 1361 & 11.70 & 23.65 & 28.64 & - & - & 29.28 & - \\
\hline Phenylethyl alcohol & 1903 & - & 0.63 & - & 0.35 & 0.54 & - & - \\
\hline Total & & 11.70 & 56.38 & 63.14 & 80.89 & 76.06 & 53.06 & \\
\hline \multicolumn{9}{|l|}{ Aldehydes } \\
\hline Hexanal & 1081 & 29.01 & 5.43 & 7.92 & 6.31 & 5.33 & 6.68 & Green \\
\hline Furfural & 1460 & 21.59 & - & - & - & - & - & - \\
\hline Benzaldehyde & 1515 & 7.95 & 1.90 & 1.30 & 3.42 & 1.66 & 1.27 & Bitter almond \\
\hline Total & & 58.55 & 7.33 & 9.22 & 9.73 & 6.99 & 7.95 & \\
\hline \multicolumn{9}{|l|}{ Ester } \\
\hline Acetic acid (hexyl ester) & 1265 & - & 2.28 & 3.48 & 1.76 & 3.01 & 4.26 & - \\
\hline Total & & 0.00 & 2.28 & 3.48 & 1.76 & 3.01 & 4.26 & \\
\hline \multicolumn{9}{|l|}{ Acid } \\
\hline Acetic acid & 1449 & 5.57 & 21.33 & 13.58 & - & 6.28 & 16.47 & Vinegar, acid \\
\hline Total & & 5.57 & 21.33 & 13.58 & 0.00 & 6.28 & 16.47 & \\
\hline \multicolumn{9}{|l|}{ Others } \\
\hline 2-Pentylfuran & 1231 & 24.18 & 10.47 & 9.62 & 6.10 & 6.07 & 16.97 & Fruity, green \\
\hline Naphthalene & 1740 & - & 2.21 & 0.95 & 1.52 & 1.60 & 1.28 & Camphoric \\
\hline Total & & 24.18 & 12.68 & 10.57 & 7.62 & 7.67 & 18.25 & \\
\hline
\end{tabular}

${ }^{\mathrm{a}}$ Retention indices on DB-Wax column; ${ }^{\mathrm{b}}$ Odor perceived at the sniffing port

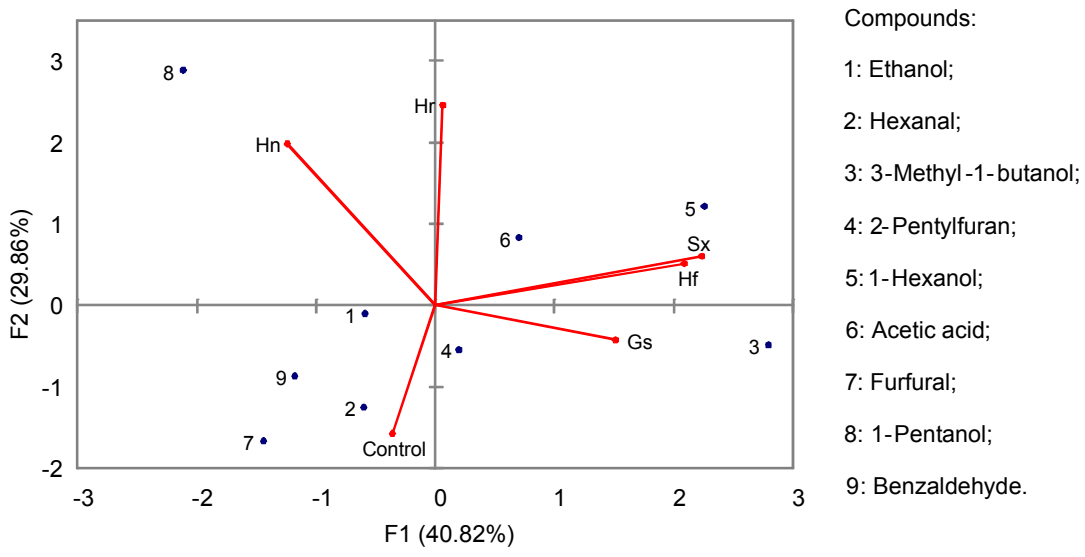

Fig. 4 Principal component analysis of volatiles of traditional sourdough steamed bread obtained by P\&T method

$53.06 \%$ to $80.89 \%$ in five samples. Among the alcohols, ethanol, which is considered as the key compound produced by yeasts and $\mathrm{LAB}$, proved to be the most dominant in all samples. Acetic acid (hexyl ester) was detected in sourdough bread samples, although in a lower relative proportion as compared to other compounds. Acetic acid (hexyl ester) was not previously reported in sourdough fermented breads. The presence of low amount of acetic acid (hexyl ester) is probably due to evaporation during steaming process. 


\subsection{Comparison of three techniques}

Extraction of volatiles is the most critical step in the identification odorants. Limitations of different extraction methods may lead to a biased picture of the overall aroma profile of any food. A description of considerable variation in the aroma groups obtained by different methods has been a key point of many previous studies (Caldeira et al., 2007; Wu et al., 2012; Xia et al., 2014). In this study, three methods were applied to analyze the volatiles in steamed bread made by sourdough. All three methods provided a diversity of compounds, making each method unique (Fig. 4). The results showed that 19 compounds were extracted by SDE, of which the aldehyde group (12) proved to be the dominant one among all the odorants extracted by this method (Table 1). Similarly, 20 compounds were detected by SPME, and most of them were aldehydes ( 7 compounds) and alcohols (7 compounds), whereas only 12 compounds were detected by P\&T, and most of them were identified as alcohols (5 compounds) (Tables 1 and 2).

Combing multiple techniques is a way of providing more individual advantages, but they also suffer from methodological limitations. SDE is one of the most popular extraction techniques for the isolation of volatile flavor components (Chaintreau, 2001). It can extract the aroma components with high and low molecular mass and low volatility. However, the major issues with SDE are the low recovery of compounds having high volatility, loss of analytes as artifacts are generated, as well as oxidation and thermal degradation. For example, some thermolabile volatile compounds can break down under the influence of high temperature. Siegmund et al. (1997) reported the generation of an artifact, 5,6-dihydro-2,4,6trimethyl-4H-1,3,5-dithiazine, while using SDE extraction. Ethanol and acetic acid are important aromatic compounds in steamed breads (Kim et al., 2009; $\mathrm{Wu}$ et al., 2012), but these are highly volatile and therefore could not be detected by SDE.

SPME and P\&T are rather mild methods for the isolation of volatiles without the risk of artifact formation. However, they are not suitable for the isolation of high molecular weight compounds, such as 1-octen-3-ol, $(E, E)$-2,4-decadienal, and (E)-2-nonenal, which are the important aroma compounds in steamed bread (Vermeulen et al., 2007).

\section{Conclusions}

For the first time, the volatile aroma profile of CSBs made by traditional sourdoughs has been explored using three different methods of aroma extraction. The results corroborate and extend the previous findings. Apart from the different microbial profiles, it is important to look into several other factors that may contribute toward the aroma development in steamed breads. The combined use of different extraction methods can provide better insights into the aroma profile of any product. It is important to mention that, though GC-MS is considered a powerful tool for the identification of volatiles or the compounds able to volatilize through derivation, the identification based on GC-MS faces many challenges like the identification of many isomers and isobars of the volatile compounds, such as lipids. So, there is a need to couple GC with other sensitive mass analysis techniques such as time-of-flight (TOF), a technique providing very high resolution (Tachibana, 2014). The superior performance of two-dimensional GC coupled to TOF $(\mathrm{GC} \times \mathrm{GC} / \mathrm{TOF}-\mathrm{MS})$ has been demonstrated (Yao et al., 2015).

\section{Acknowledgements}

We sincerely thank Dr. Bao-guo SUN, Dr. Huan-lu SONG, and Dr. Bei WANG (Beijing Technology and Business University (BTBU), China) for providing GC-O-MS analysis for this study.

\section{Compliance with ethics guidelines}

Guo-hua ZHANG, Tao WU, Faizan A. SADIQ, Huan-yi YANG, Tong-jie LIU, Hui RUAN, and Guo-qing HE declare that they have no conclict of interest.

This article does not contain any studies with human or animal subjects performed by the any of the authors.

\section{References}

Arendt, E.K., Ryan, L.A.M., Bello, F.D., 2007. Impact of sourdough on the texture of bread. Food Microbiol., 24(2):165-174. http://dx.doi.org/10.1016/j.fm.2006.07.011

Caldeira, M., Rodrigues, F., Perestrelo, R., et al., 2007. Comparison of two extraction methods for evaluation of volatile constituents patterns in commercial whiskeys. Elucidation of the main odour-active compounds. Talanta, 74(1):78-90.

http://dx.doi.org/10.1016/j.talanta.2007.05.029

Chaintreau, A., 2001. Simultaneous distillation-extraction: 
from birth to maturity—review. Flavour Frag. J., 16(2): 136-148. http://dx.doi.org/10.1002/ffj.967

Damiani, P., Gobbetti, M., Cossignani, L., et al., 1996. The sourdough microflora. Characterization of hetero- and homofermentative lactic acid bacteria, yeasts and their interactions on the basis of the volatile compounds produced. LWT-Food Sci. Technol., 29(1-2):63-70.

http://dx.doi.org/10.1006/fstl.1996.0009

Etievant, P.X., 1996. Artifacts and contaminants in the analysis of food flavor. Crit. Rev. Food Sci. Nutr., 36(7):733-745. http://dx.doi.org/10.1080/10408399609527746

Frankel, E.N., 1982. Volatile lipid oxidation products. Prog. Lipids Res., 22:4-33.

Frasse, P., Lambert, S., Richard-Molard, D., et al., 1993. The influence of fermentation on volatile compounds in French bread dough. LWT-Food Sci. Technol., 26(2): 126-132. http://dx.doi.org/10.1006/fstl.1993.1027

Galle, S., Arendt, E.K., 2014. Exopolysaccharides from sourdough lactic acid bacteria. Crit. Rev. Food Sci. Nutr., 54(7):891-901. http://dx.doi.org/10.1080/10408398.2011.617474

Gobbetti, M., Simonetti, M.S., Corsetti, A., et al., 1995. Volatile compound and organic acid productions by mixed wheat sour dough starters: influence of fermentation parameters and dynamics during baking. Food Microbiol., 12:497-507. http://dx.doi.org/10.1016/S0740-0020(95)80134-0

Hansen, A., Schieberle, P., 2005. Generation of aroma compounds during sourdough fermentation: applied and fundamental aspects. Trends Food Sci. Technol., 16(1-3): 85-94.

http://dx.doi.org/10.1016/j.tifs.2004.03.007

Hansen, B., Hansen, A., 1994. Volatile compounds in wheat sourdoughs produced by lactic acid bacteria and sourdough yeasts. Zeitschrift für Lebensmittel-Untersuchung und-Forschung, 198(3):202-209. http://dx.doi.org/10.1007/BF01192596

He, Z.H., Liu, A.H., Peña, R.J., et al., 2003. Suitability of Chinese wheat cultivars for production of northern style Chinese steamed bread. Euphytica, 131(2):155-163. http://dx.doi.org/10.1023/A:1023929513167

Kam, W.Y., Wan, A.W.M., Sahilah, A.M., et al., 2011. Volatile compounds and lactic acid bacteria in spontaneous fermented sourdough. Sains Malays., 40(2):135-138.

Kang, K.M., Baek, H.H., 2014. Aroma quality assessment of Korean fermented red pepper paste (gochujang) by aroma extract dilution analysis and headspace solid-phase microextraction-gas chromatography-olfactometry. Food Chem., 145(4):488-495.

http://dx.doi.org/10.1016/j.foodchem.2013.08.087

Katina, K., Poutanen, K., 2013. Nutritional Aspects of Cereal Fermentation with Lactic Acid Bacteria and Yeast. In: Gobbetti, M., Gänzle, M. (Eds.), Handbook on Sourdough Biotechnology. Springer US, p.229-244.

Kim, Y., Huang, W., Zhu, H., et al., 2009. Spontaneous sourdough processing of Chinese northern-style steamed breads and their volatile compounds. Food Chem., 114(2): 685-692.

http://dx.doi.org/10.1016/j.foodchem.2008.10.008

Liao, Y., Miller, R.A., Hoseney, R.C., et al., 1998. Role of hydrogen peroxide produced by baker's yeast on dough rheology 1. Cereal Chem., 75(5):612-616. http://dx.doi.org/10.1094/CCHEM.1998.75.5.612

Lorenz, K., Kulp, K., 2003. Handbook of Dough Fermentations. CRC Press, Boca Raton, p.110-127.

Luangsakul, N., Keeratipibul, S., Jindamorakot, S., et al., 2009. Lactic acid bacteria and yeasts isolated from the starter doughs for Chinese steamed buns in Thailand. LWTFood Sci. Technol., 42(8):1404-1412. http://dx.doi.org/10.1016/j.lwt.2009.03.007

Mahadevan, K., Farmer, L., 2006. Key odor impact compounds in three yeast extract pastes. J. Agric. Food Chem., 54(19):7242-7250. http://dx.doi.org/10.1021/jf061102x

Mamede, M.E.O., Pastore, G.M., 2006. Study of methods for the extraction of volatile compounds from fermented grape must. Food Chem., 96(4):586-590. http://dx.doi.org/10.1016/j.foodchem.2005.03.013

Mann, D., Morrison, W.R., 1975. Effects of ingredients on the oxidation of linoleic acid by lipoxygenase in bread doughs. J. Sci. Food Agric., 26(4):493. http://dx.doi.org/10.1002/jsfa.2740260415

Martínez-Anaya, M.A., Llin, M.L., Macías, M.P., et al., 1994. Regulation of acetic acid production by homo- and heterofermentative lactobacilli in whole-wheat sour-doughs. Zeitschrift für Lebensmittel-Untersuchung und-Forschung, 199(3):186-190. http://dx.doi.org/10.1007/BF01193440

Plessas, S., Bekatorou, A., Gallanagh, J., et al., 2008. Evolution of aroma volatiles during storage of sourdough breads made by mixed cultures of Kluyveromyces marxianus and Lactobacillus delbrueckii ssp. bulgaricus or Lactobacillus helveticus. Food Chem., 107(2):883-889. http://dx.doi.org/10.1016/j.foodchem.2007.09.010

Reineccius, G., 2005. Flavor Chemistry and Technology, 2nd Ed. Boca Raton, p.43-66.

Rosenquist, H., Hansen, H., 1998. The antimicrobial effects of organic acids, sourdough and nisin against Bacillus subtilis and B. licheniformis isolated from wheat bread. $J$. Appl. Microbiol., 85(3):621-631. http://dx.doi.org/10.1046/j.1365-2672.1998.853540.x

Salim-ur-Rehman, Paterson, A., Piggott, J.R., 2006. Flavour in sourdough breads: a review. Trends Food Sci. Technol., 17(10):557-566. http://dx.doi.org/10.1016/j.tifs.2006.03.006

Schieberle, P., 1996. Intense aroma compounds-useful tools to monitor the influence of processing and storage on bread aroma. Adv. Food Sci. (CMTL), 18:237-244.

Siegmund, B., Leitner, E., Mayer, I., et al., 1997. 5,6-Dihydro 2,4,6-trimethyl-4H-1,3,5-dithiazine - an aroma-active compound formed in course of the likens-nickerson extraction. Zeitschrift für Lebensmittel-Untersuchung und-Forschung, 
205(1):73-75.

http://dx.doi.org/10.1007/s002170050126

Spicher, G., Rabe, E., Sommer, R., et al., 1982. Communication: on the behavior of heterofermentative sourdough bacteria and yeasts in mixed culture. Zeitschrift für Lebensmittel-Untersuchung und-Forschung, 174(3):222-227. http://dx.doi.org/10.1007/BF01079978

Starr, G., Hansen, Å.S., Petersen, M.A., et al., 2015. Aroma of wheat porridge and bread-crumb is influenced by the wheat variety. LWT-Food Sci. Technol., 63(1):590-598. http://dx.doi.org/10.1016/j.lwt.2015.03.048

Stolz, P., Böcker, G., Vogel, R.F., et al., 1993. Utilization of maltose and glucose by lactobacilli isolated from sourdough. FEMS Microbiol. Lett., 109(2-3):237-242. http://dx.doi.org/10.1111/j.1574-6968.1993.tb06174.x

Tachibana, C., 2014. What's next in 'omics: the metabolome. Science, 345(6203):1519-1521. http://dx.doi.org/10.1126/science.345.6203.1519

Vas, G., Vékey, K., 2004. Solid-phase microextraction: a powerful sample preparation tool prior to mass spectrometric analysis. J. Mass Spectrom., 39(3):233-254. http://dx.doi.org/10.1002/jms.606

van den Dool, H., Kratz, P.D., 1963. A generalization of the retention index system including line temperature programmed gas liquid partition chromatography. J. Chromatogr. A, 11(C):463-471. http://dx.doi.org/10.1016/S0021-9673(01)80947-X

Vermeulen, N., Czerny, M., Gänzle, M.G., et al., 2007. Reduction of $(E)$-2-nonenal and $(E, E)$-2,4-decadienal during sourdough fermentation. J. Cereal Sci., 45(1):78-87. http://dx.doi.org/10.1016/j.jcs.2006.07.002

Wang, R., Yang, C., Song, H.L., 2012. Key meat flavour compounds formation mechanism in a glutathione-xylose Maillard reaction. Food Chem., 131(1):280-285. http://dx.doi.org/10.1016/j.foodchem.2011.08.079

Wu, C., Liu, R., Huang, W., et al., 2012. Effect of sourdough fermentation on the quality of Chinese northern-style steamed breads. J. Cereal Sci., 56(2):127-133. http://dx.doi.org/10.1016/j.jcs.2012.03.007

Xia, Y., Zhang, F., Wang, W., et al., 2014. Analysis of volatile compounds from siraitia grosvenorii by headspace solidphase microextraction and gas chromatography-quadrupole time-of-flight mass spectrometry. J. Chromatogr. Sci., 53(1):1-7. http://dx.doi.org/10.1093/chromsci/bmu012

Yao, F., Yi, B., Shen, C., et al., 2015. Chemical analysis of the Chinese liquor Luzhoulaojiao by comprehensive twodimensional gas chromatography/time-of-flight mass spectrometry. Sci. Rep., 5:9553.

Zhang, G., He, G., 2013. Predominant bacteria diversity in Chinese traditional sourdough. J. Food Sci., 78(8): M1218-M1223.

http://dx.doi.org/10.1111/1750-3841.12193

Zhang, G., Sadiq, F.A., Zhu, L., et al., 2015. Investigation of microbial communities of Chinese sourdoughs using culture-dependent and DGGE approaches. J. Food Sci., 80(11):M2535-M2542. http://dx.doi.org/10.1111/1750-3841.13093

Zhang, J., Liu, W., Sun, Z., et al., 2011. Diversity of lactic acid bacteria and yeasts in traditional sourdoughs collected from western region in Inner Mongolia of China. Food Control, 22(5):767-774.

http://dx.doi.org/10.1016/j.foodcont.2010.11.012

\section{中文概要}

题 目: 传统酸面团制备的馒头特征风味物质的研究

目 的: 通过同时蒸馏萃取 (SDE)、顶空固相微萃取 (SPME) 及吹扫捕集 (P\&T) 技术结合气相色 谱-嗅闻-质谱（GC-O-MS）技术对传统酸面团制 备的馒头特征风味成分进行分析, 并初步探讨特 征风味形成机理。

创新点: 首次采用不同风味物质分析技术探索传统酸面团 制备的馒头关键风味成分。

方 法: 采用同时 SDE、SPME 及 P\&T 方法, 对不同传统 酸面团制备的馒头特征风味物质进行分析。

结 论: SDE 结果显示, 反式-2-壬烯醛和反式-2,4-癸二烯 醛为传统酸面团馒头中关键风味成分。SPME 和 P\&T 方法表明乙醇和醋酸是关键风味物质。因 此, 需要结合不同技术方法, 全面分析传统酸面 团制备的馒头中特征风味成分。

关键词: 馒头; 酸面团; 同时蒸馏萃取; 固相微萃取; 吹 扫捕集 